\title{
Molecular detection of Wolbachia endosymbiont in reptiles and their ectoparasites
}

\author{
Ranju Ravindran Santhakumari Manoj ${ }^{1} \cdot$ Maria Stefania Latrofa ${ }^{1} \cdot$ Jairo Alfonso Mendoza-Roldan ${ }^{1}$. \\ Domenico Otranto ${ }^{1,2}$ (I)
}

Received: 21 May 2021 / Accepted: 30 June 2021

(c) The Author(s) 2021

\begin{abstract}
Wolbachia, a maternally transmitted Gram-negative endosymbiont of onchocercid nematodes and arthropods, has a role in the biology of their host; thus it has been exploited for the filariasis treatment in humans. To assess the presence and prevalence of this endosymbiont in reptiles and their ectoparasites, blood and tail tissue as well as ticks and mites collected from them were molecularly screened for Wolbachia DNA using two sets of primers targeting partial 16S rRNA and Wolbachia surface protein (wsp) genes. Positive samples were screened for the partial 12S rRNA and cytochrome $c$ oxidase subunit 1 (cox1) genes for filarioids. Of the different species of lizards (Podarcis siculus, Podarcis muralis and Lacerta bilineata) and snakes (Elaphe quatuorlineata and Boa constrictor constrictor) screened from three collection sites, only P. siculus scored positive for Wolbachia 16S rRNA. Among ectoparasites collected from reptiles (Ixodes ricinus ticks and Neotrombicula autumnalis, Ophionyssus sauracum and Ophionyssus natricis mites), I. ricinus ( $n=4 ; 2.8 \%$; 95\% CI, 0.9-7) from P. siculus, N. autumnalis ( $n=2$ each; 2.8\%; 95\% CI, 0.9-6.5) from P. siculus and P. muralis and O. natricis $(n=1 ; 14.3 \%$; 95\% CI, 0.7-55.4) from Boa constrictor constrictor scored positive for Wolbachia DNA. None of the positive Wolbachia samples scored positive for filarioids. This represents the first report of Wolbachia in reptilian hosts and their ectoparasites, which follows a single identification in the intestinal cells of a filarioid associated with a gecko. This data could contribute to better understand the reptile filarioid-Wolbachia association and to unveil the evolutionary pattern of Wolbachia in its filarial host.
\end{abstract}

Keywords Wolbachia $\cdot$ Lizard $\cdot$ Ixodes ricinus $\cdot$ Neotrombicula autumnalis $\cdot$ Snake

\section{Introduction}

Wolbachia, an obligate intracellular endosymbiont of arthropods and filarioids, has gained more attention in the scientific community due to its role in the biology of filarioids and their vectors and its contribution in the development of immunopathology of filariasis (Bandi et al. 1998; Taylor, 2003). Wolbachia pipientis represent the single species of the genus Wolbachia with supergroups used to classify the large genetic diversity of different strains identified

Handling Editor: Julia Walochnik

Domenico Otranto

domenico.otranto@uniba.it

1 Department of Veterinary Medicine, University of Bari, Valenzano, Italy

2 Faculty of Veterinary Sciences, Bu-Ali Sina University, Hamedan, Iran
(Casiraghi et al. 2005). This endosymbiont has been reported in around $50 \%$ of the terrestrial arthropod species which includes insects, mites, crustaceans, spiders, scorpions, collembolans and in several species of onchocercid nematodes (Ferri et al. 2011; Lefoulon et al. 2016; Manoj et al. 2021), as well as in non-filarial plant nematodes of the order Tylenchida (Haegeman et al. 2009). Onchocercid-Wolbachia association is obligatory being required for the reproduction, development and long-term survival of the host (Martin and Gavotte 2010). Meanwhile, arthropod-Wolbachia association could be considered more parasitic, since the bacteria gain fitness advantage by reproductive manipulations of the host, modifying the iron homeostasis and reducing the vector capacity of their hosts (Bandi et al. 2001; Kremer et al. 2009; Martinez et al. 2017). Based on their genetic evolution, Wolbachia have been classified as 17 supergroups, from A to S (Fenn et al. 2006; Lefoulon et al. 2020a, b), in which Wolbachia of arthropods were included in supergroups A, $\mathrm{B}, \mathrm{E}, \mathrm{H}, \mathrm{I}, \mathrm{K}$, that of nematodes in $\mathrm{C}, \mathrm{D}, \mathrm{J}$ (Glowska et al. 
2015) and plant-parasitic nematodes in L (Brown et al. 2016). Among these supergroups, $F$ is an exception being reported in both arthropods (i.e. termites, spiders, mites, bugs; Rasgon and Scott 2004; Ros et al. 2009) and onchocercid nematodes (i.e., human filariae, Mansonella; filariae of black bear, Cercopithifilaria japonica; filariae of geckoes, Madathamugadia hiepei; Casiraghi et al. 2001; Casiraghi et al. 2005; Keiser et al. 2008; Ferri et al. 2011; Lefoulon et al. 2012). For long time, Wolbachia was considered to be present only in two of the eight onchocercid subfamilies, i.e. Onchocercinae and Dirofilariinae (Lefoulon et al. 2012). However, the discovery of this bacterium in the filariae of geckoes (i.e. Splendidofilariinae) suggested that Wolbachia diverged before the first bacterial invasion in onchocercid lineage (Bandi et al. 1998; Fenn et al. 2006; Casiraghi et al. 2004). Moreover, previous inferences about the existence of a congruency between Wolbachia and the filarial host phylogenies (Bandi et al. 1998) has become inaccurate after the addition of supergroup F (Lefoulon et al. 2012). Though Wolbachia was detected once in filarial worms from geckoes (i.e., M. hiepei), its presence in the reptilian species is not known. Hence the current study aimed to assess the presence and prevalence of Wolbachia in synanthropic lizards and snakes and their ectoparasites from southern Italy.

\section{Materials and methods}

\section{Sample collection}

The reptiles and ectoparasites collected under the frame of previous studies (Mendoza-Roldan et al. 2019, 2021a) were included in the study. Specimens were collected from two areas of the Apulia region (site 1, a peri-urban region in Bari and site 2, a zoological park in Fasano), and from Basilicata region (site 3) in South Italy. From site 1, 38 mite infested lizards, and from site 3, 128 lizards and snakes were captured (Mendoza-Roldan et al. 2019). Reptile species were identified by using specific reference keys (Arnold, 2002). Blood samples were collected in EDTA vials via tail fracture/cardiocentesis and from the ventral coccygeal vein in case of lizards and snakes, respectively, and stored at $-20{ }^{\circ} \mathrm{C}$ until further processing. A small portion of the tail tissue was also collected from lizards and stored in $70 \%$ ethanol until DNA isolation.

\section{DNA isolation, molecular and sequencing analyses}

Genomic DNA from blood of reptiles and tail tissues of lizards were extracted using Qiagen blood Mini kit and Qiagen DNeasy Blood \& Tissue kit (Qiagen, Hilden, Germany), respectively, following the manufacturer's instructions, whilst guanidine isothiocyanate protocol (GT)
(Chomkzynski, 1993) was used to extract the DNA from ticks and mites, and the elutions were made in $50 \mu \mathrm{AE}$ buffer. All samples were tested molecularly for the endosymbiont, Wolbachia using two sets of primers targeting partial 16S rRNA and Wolbachia surface protein (wsp) genes and the positive samples were screened for the partial 12S rRNA and cytochrome $c$ oxidase subunit $1(\cos 1)$ genes for filarioids (Casiraghi et al. 2004; Martin et al. 2005; Otranto et al. 2011; Zhou et al. 1998). All amplified PCR products were visualized in $2 \%$ agarose gel containing Gel Red ${ }^{\circledR}$ nucleic acid gel stain (VWR International PBI, Milan, Italy) and documented in Gel Logic 100 (Kodak, NY, USA). The PCR products were purified and sequenced in both directions using the same primers, employing the Big Dye Terminator v.3.1 chemistry in a 3130 Genetic analyzer (Applied Biosystems, CA, USA) in an automated sequencer (ABI-PRISM 377). Nucleotide sequences were edited aligned and analysed using BioEdit and compared with available sequences in the GenBank using Basic Local Alignment Search Tool (BLAST; http://blast.ncbi.nlm.nih.gov/Blast.cgi).

\section{Phylogenetic analyses}

Representative sequences of the 16S rRNA of Wolbachia were included along with the sequences available in the GenBank database for phylogenetic analyses. Phylogenetic relationships were inferred using maximum likelihood (ML) method based on Kimura 2-parameter model (Kimura, 1980) with discrete Gamma distribution $(+G)$ to model evolutionary rate differences among sites selected by best-fit model (Kumar et al. 2018). Evolutionary analyses were conducted on 1000 bootstrap replications using the MEGA X software (Tamura et al. 2013). Homologous sequences from Ehrlichia ruminantium and Ehrlichia chaffeensis were used as outgroups (NR074513, AF147752).

\section{Statistical analysis}

Prevalence of Wolbachia infection among reptiles (proportion of reptiles and ectoparasites infected by Wolbachia on the total population of reptiles and ectoparasites collected from different sites) was assessed. Statistical analysis was done using StatLib software. Exact binomial 95\% confidence intervals (CI) were established for proportions.

\section{Results}

Of the different species of lizards (Podarcis siculus, Podarcis muralis and Lacerta bilineata) and snakes (Elaphe quatuorlineata and Boa constrictor constrictor), only the blood $(\mathrm{n}=5,3.4 \% ; 95 \%$ CI, 1.37-7.8) and tissue samples $(\mathrm{n}=3$, $2.1 \%$; 95\% CI, 0.6-6.1) of P. siculus scored positive for 


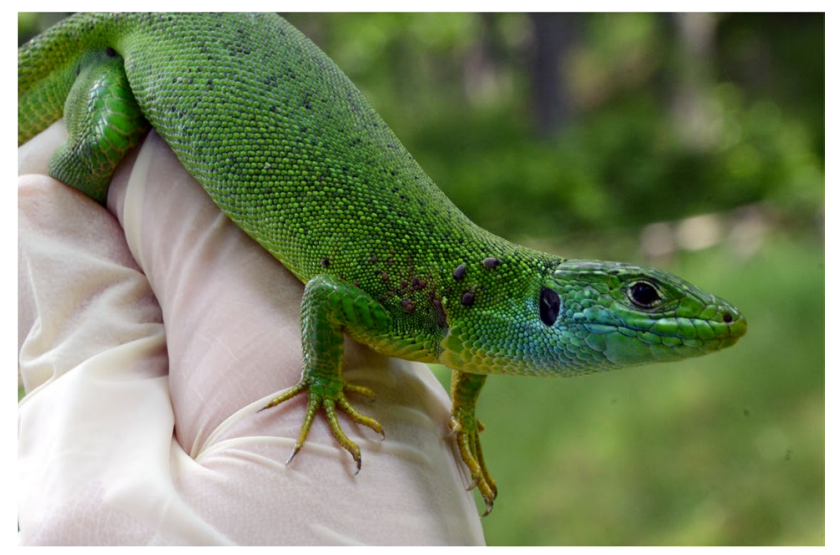

Fig. 1 Lacerta bilineata infested with Ixodes ricinus

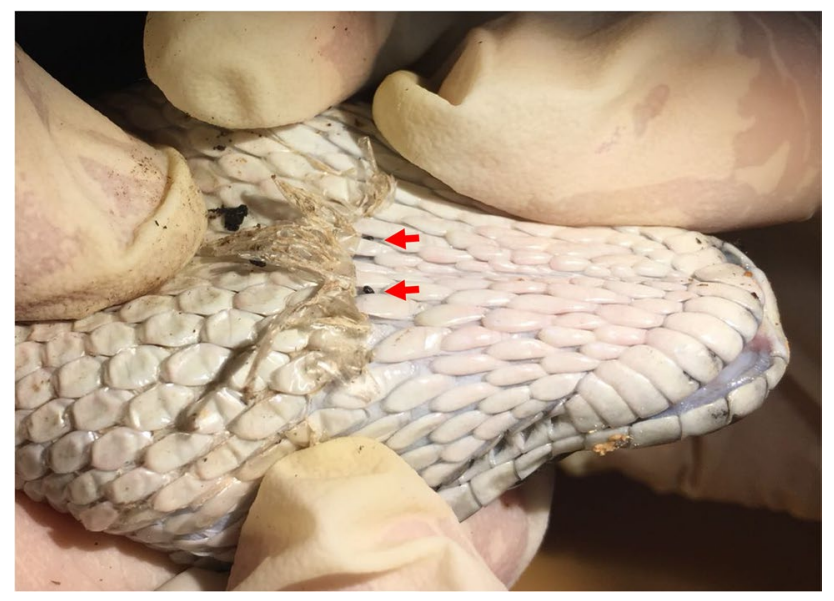

Fig. 2 Boa constrictor constrictor infested with Ophionyssus natricis (red arrows)

Wolbachia 16S rRNA. Of the 5 Wolbachia positive blood samples, 2 were collected from site 1 and 3 from site 3 , whilst all positive tissue samples were collected from site 3. Among ectoparasites collected from reptiles (Ixodes ricinus tick and Neotrombicula autumnalis, Ophionyssus sauracum and Ophionyssus natricis mites) (Figs. 1,2), I. ricinus $(\mathrm{n}=4 ; 2.8 \%$; 95\% CI, 0.9-7) from P. siculus, $N$. autumnalis ( $\mathrm{n}=2$ each; 2.8\%; 95\% CI, 0.9-6.5) from P. siculus and $P$. muralis and $O$. natricis $(\mathrm{n}=1 ; 14.3 \%$; 95\% CI, 0.7-55.4) from Boa constrictor constrictor, scored positive for Wolbachia. In particular, positive ticks were collected from both site $1(\mathrm{n}=1,2.6 \%$; 95\% CI, 0.1-13.3) and $3(\mathrm{n}=3$, $2.5 \%$; 95\% CI, 0.7-7.4), while positive mites were collected from sites $2(\mathrm{n}=1,33.3 \%$; 95\% CI, 1.7-86.5) and $3(\mathrm{n}=4$, $3.4 \%$; 95\% CI, 1.2-8.3). The lizard and ectoparasite samples collected from site 2 had the highest prevalence (33.3\%) of Wolbachia followed by site $3(5.9 \%)$ and site $1(2.3 \%)$. Corresponding reptilian hosts of the ectoparasites positive for Wolbachia DNA were negative for this endosymbiont.
PCR products of wsp gene had non-specific amplifications, whereas none of 16S rRNA positive Wolbachia samples scored positive for filarioids.

Phylogenetic analysis of the $16 \mathrm{~S}$ rRNA gene yielded strict consensus in overlapping the topology of the trees, with a strong bootstrap value (up to 100\%) for each supergroup clade (Fig. 3). All sequences of Wolbachia detected in lizard and reptile ectoparasites were clustered in two monophyletic clades (bootstrap value of $84 \%$ ) and as sister clade of that of Wolbachia supergroup B. Representative sequences of Wolbachia sequences were deposited in GenBank (accession numbers: MZ490470-MZ490476).

\section{Discussion}

The current study assessed the presence of Wolbachia in tail tissues and blood of lizards suggesting its circulation in these host species.

Though the presence of this bacterium has been previously reported in the intestinal cells of the saurian filarioid M. hiepei (Lefoulon et al. 2012), it has never been identified in reptilian hosts and/or their ectoparasites. In filarioids this endosymbiont is usually located in hypodermal lateral chords (Taylor and Hoerauf 1999; Kramer et al. 2003). Previous studies suggested that the release of Wolbachia into the blood occurs following the death or damage of the worms after anti-filarial chemotherapy (Taylor 2003). In the current study, though the presence of this bacterium was detected in the reptilian host, none of them were positive for any filarioid. This suggests that the associated filarioids and their larval stages may be located in tissues other than tail or blood. Filarial nematodes belonging to the genera Foleyella, Macdonaldius, Splendinofilaria and Oswaldofilaria are found to infect reptiles vectored by mosquitoes (MendozaRoldan et al. 2021b). Most of them are found in extra intestinal sites such as the lungs, circulatory system and subcutaneous tissue (Jacobson et al. 2020). It has been also postulated that the presence of blood circulating Wolbachia antigens might result from the natural excretion of Wolbachia products by the nematodes or the release of these products from the dying worms (Shiny et al. 2009; Anuradha et al. 2012). Similarly, immunohistology studies in human onchosarcoma and blood cells have suggested free endobacteria outside of the filariae in host's tissues (Brattig et al. 2004) which further strengthens the possibility of finding the Wolbachia in host blood and tissues. Indeed, the finding of Wolbachia in reptiles opens the possibility of widening the knowledge about the evolution of this endobacterium.

The presence of Wolbachia in mites of the species $N$. autumnalis and $O$. natricis is new to the scientific community and could be of importance considering the biodiversity in trombiculid and macronyssid mites from reptiles 
Fig. 3 Phylogenetic relationship of Wolbachia detected in this study (in bold) and other available from GenBank belonging to different supergroups based on a partial sequence of the 16S rRNA gene. Evolutionary analysis was conducted on 1000 bootstrap replications using Maximum Likelihood method and Kimura 2-parameter model with discrete Gamma distribution $(+G)$ to model evolutionary rate differences among sites selected by best-fit model. GenBank accession number and host species are indicated

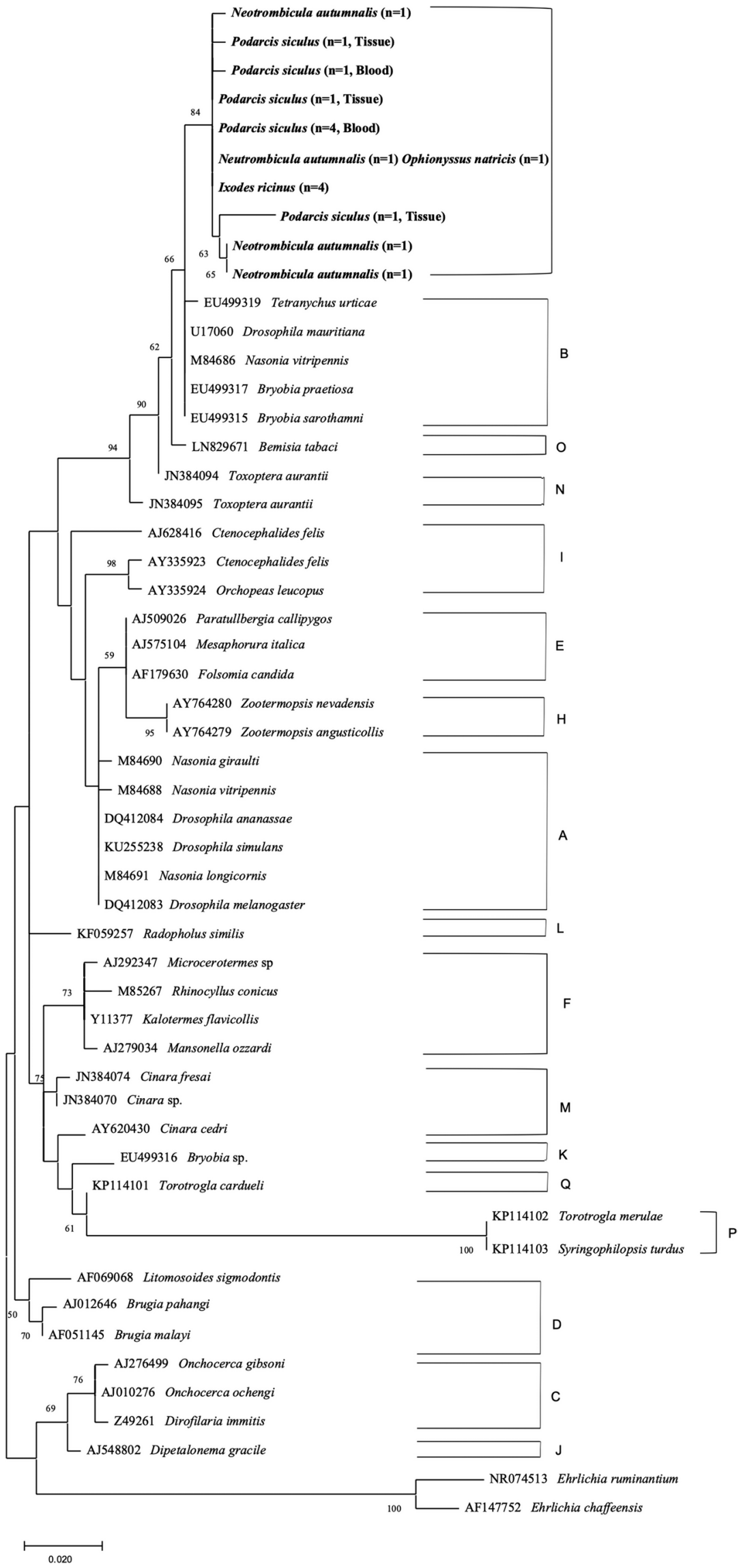


(Mendoza-Roldan et al. 2020; Bezerra-Santos et al. 2021). In trombiculid mite species, only larval stages are parasitic and the infestations are self-limiting (Bassini-Silva et al. 2017; Jacinavicius et al. 2018), which reduces the possibility of translocation of the mite from an infected to an uninfected host. The finding of Wolbachia positive mites from the endosymbiont negative reptiles may indicate a vertical transmission of this bacterium. Conversely, it was suggested that presence of Wolbachia in the hypodermal cells of onchocercid nematodes is an ancestral somatic tissue-preference, which may aid the horizontal transmission (Landmann et al. 2012; Lefoulon et al. 2012). The latter transmission pattern could also be explained by Wolbachia natural excretion or as a consequence of dying filarial worms releasing endobacteria in host's tissues and blood (Brattig et al. 2004; Shiny et al. 2009; Anuradha et al. 2012). The finding of infected I. ricinus on uninfected host can be explained by the translocation of this ectoparasite while feeding (i.e. from an infected host to an uninfected host). However, the natural occurrence of this bacterium in ticks and mites cannot be ruled out, given the high prevalence (i.e. from 20 to around $75 \%$ ) reported amongst arthropods (Werren et al. 1995; Jeyaprakash and Hoy 2000). Phylogenetic relationship of Wolbachia from reptiles and its ectoparasites with supergroup B suggests the possibility of a horizontal transfer of this bacterium from the host to the ectoparasites or vice versa. Moreover, the low genetic divergence in supergroup $\mathrm{F}$ when compared to supergroups $\mathrm{C}$ and $\mathrm{D}$ and a close resemblance with supergroups $\mathrm{B}$ and $\mathrm{A}$ also supports the hypothesis that horizontal transmission has occurred between Onchocercidae and arthropods (Lefoulon et al. 2012).

\section{Conclusion}

Being firstly reported from the reptilian host and their ectoparasites, a detailed investigation is needed to understand the relationship of this reptilian Wolbachia and the associated filarial host. The new findings may further complement the evolutionary picture of Wolbachia and its filarial host. Hence, detailed research in this area will help to understand the tropism of this endosymbiont in its reptilian filarioid host and to unravel the evolutionary traits of this enigmatic bacterium.

Author contribution RRSM, DO and MSL conceived the study. JAMR and DO performed field work and collection of samples. RRSM performed laboratory work and analyzed data. RRSM performed phylogenetic analysis. RRSM wrote the first draft of the manuscript. RRSM, MSL, JAMR and DO reviewed and wrote the final draft of the manuscript. All authors read and approved the final version of the manuscript.
Funding Open access funding provided by Università degli Studi di Bari Aldo Moro within the CRUI-CARE Agreement.

\section{Declarations}

Ethics approval The study was conducted in accordance with ethical principles (Declaration of Helsinki), written informed consent was obtained from the participants, and the research protocol was approved by the ethics committee of the University Hospital of Bari (n. 6394, prot. n., 0,044,469-23,062,020). Protocols for lizard collection and sampling were approved by the Commission for Bioethics and Animal Welfare of the Department of Veterinary Medicine of the University of Bari and authorized by the Ministry for Environment, Land and Sea Protection of Italy (approval number 0016973/2018), the Societas Herpetologica Italica (approval number SHI-aut-ER-12-2018) and the "Istituto Superiore per la Protezione e la Ricerca Ambientale" (approval number 41180).

Competing interests The authors declare no competing interests.

Open Access This article is licensed under a Creative Commons Attribution 4.0 International License, which permits use, sharing, adaptation, distribution and reproduction in any medium or format, as long as you give appropriate credit to the original author(s) and the source, provide a link to the Creative Commons licence, and indicate if changes were made. The images or other third party material in this article are included in the article's Creative Commons licence, unless indicated otherwise in a credit line to the material. If material is not included in the article's Creative Commons licence and your intended use is not permitted by statutory regulation or exceeds the permitted use, you will need to obtain permission directly from the copyright holder. To view a copy of this licence, visit http://creativecommons.org/licenses/by/4.0/.

\section{References}

Anuradha R, George PJ, Kumar NP, Fay MP, Kumaraswami V, Nutman TB, Babu S (2012) Circulating microbial products and acute phase proteins as markers of pathogenesis in lymphatic filarial disease. PLoS Pathog 8(6):e1002749

Bandi C, Anderson TJC, Genchi C, Blaxter ML (1998) Phylogeny of Wolbachia pipientis in filarial nematodes. Proc R Soc Lond B 265:2407-2413

Bandi C, Trees AJ, Brattig NW (2001) Wolbachia in filarial nematodes: evolutionary aspects and implications for the pathogenesis and treatment of filarial diseases. Vet Parasitol 98:215-238

Bassini-Silva R, Jacinavicius FC, Mendoza-Roldan JA, Daemon E, Barros-Battesti DM (2017) Description of Blankaartia shatrovi $\mathrm{n}$. sp. (Acari: Trombiculidae) from Brazil. J Med Entomol 54(1):82-90

Bezerra-Santos MA, Mendoza-Roldan JA, Thompson RA, Dantas-Torres F, Otranto D (2021) Illegal wildlife trade: a gateway to zoonotic infectious diseases. Trends in Parasitology 37(3):181-184

Brattig NW, Bazzocchi C, Kirschning CJ, Reiling N, Büttner DW, Ceciliani F, Geisinger F, Hochrein H, Ernst M, Wagner H, Bandi C (2004) The major surface protein of Wolbachia endosymbionts in filarial nematodes elicits immune responses through TLR2 and TLR4. J Immunol 173(1):437-445

Brown AM, Wasala SK, Howe DK, Peetz AB, Zasada IA, Denver DR (2016) Genomic evidence for plant-parasitic nematodes as the earliest Wolbachia hosts. Sci Rep 6(1):1-4 
Casiraghi M, Bain O, Guerrero R, Martin C, Pocacqua V, Gardner SL, Franceschi A, Bandi C (2004) Mapping the presence of Wolbachia pipientis on the phylogeny of filarial nematodes: evidence for symbiont loss during evolution. Int J Parasitol 34(2):191-203

Casiraghi M, Bordenstein SR, Baldo L, Lo N, Beninati T, Wernegreen JJ, Werren JH, Bandi C (2005) Phylogeny of Wolbachia pipientis based on gltA, groEL and ftsZ gene sequences: clustering of arthropod and nematode symbionts in the F supergroup, and evidence for further diversity in the Wolbachia tree. Microbiology 151(12):4015-4022

Casiraghi M, Favia G, Cancrini G, Bartoloni A, Bandi C (2001) Molecular identification of Wolbachia from the filarial nematode Mansonella ozzardi. Parasitol Res 87(5):417-420

Chomczynski P (1993) A reagent for the single-step simultaneous isolation of RNA, DNA and proteins from cell and tissue samples. Biotechniques 15(3):532-534

Fenn K, Conlon C, Jones M, Quail MA, Holroyd NE, Parkhill J, Blaxter M (2006) Phylogenetic relationships of the Wolbachia of nematodes and arthropods. PLoS Pathog 2(10):e94

Ferri E, Bain O, Barbuto M, Martin C, Lo N, Uni S, Landmann F, Baccei SG, Guerrero R, de Souza Lima S, Bandi C (2011) New insights into the evolution of Wolbachia infections in filarial nematodes inferred from a large range of screened species. PloS one 6(6):e20843

Glowska E, Dragun-Damian A, Dabert M, Gerth M (2015) New Wolbachia supergroups detected in quill mites (Acari: Syringophilidae). Infect Genet Evol 30:140-146

Haegeman A, Vanholme B, Jacob J, Vandekerckhove TT, Claeys M, Borgonie G, Gheysen G (2009) An endosymbiotic bacterium in a plant-parasitic nematode: member of a new Wolbachia supergroup. Int J Parasitol 39(9):1045-1054

Jacinavicius FDC, Bassini-Silva R, Mendoza-Roldan JA, Muñoz-Leal S, Hingst-Zaher E, Ochoa R, Barros-Battesti DM (2018) A contribution to the knowledge of Quadraseta brasiliensis Goff and Gettinger, 1989 (Trombidiformes: Trombiculidae), with description of the deutonymph instar. Acarologia 58(2):442-456

Jacobson ER, Garner MM (eds) (2020) Infectious diseases and pathology of reptiles: color atlas and text, diseases and pathology of reptiles, vol 1. CRC Press. https://doi.org/10.1201/9780429155567

Jeyaprakash A, Hoy MA (2000) Long PCR improves Wolbachia DNA amplification: wsp sequences found in $76 \%$ of sixty-three arthropod species. Insect Mol Biol 9(4):393-405

Keiser PB, Coulibaly Y, Kubofcik J, Diallo AA, Klion AD, Traoré SF, Nutman TB (2008) Molecular identification of Wolbachia from the filarial nematode Mansonella perstans. Mol Biochem Parasitol 160(2):123-128

Kimura M (1980) A simple method for estimating evolutionary rates of base substitutions through comparative studies of nucleotide sequences. J Mol Evol 16(2):111-120

Kramer L, Passeri B, Corona S, Simoncini L, Casiraghi M (2003) Immunohistochemical/immunogold detection and distribution of the endosymbiont Wolbachia of Dirofilaria immitis and Brugia pahangi using a polyclonal antiserum raised against WSP (Wolbachia surface protein). Parasitol Res 89(5):381-386

Kremer N, Voronin D, Charif D, Mavingui P, Mollereau B, Vavre F (2009) Wolbachia interferes with ferritin expression and iron metabolism in insects. PLoS Pathog. 5(10):e1000630

Kumar S, Stecher G, Li M, Knyaz C, Tamura K (2018) MEGA X: molecular evolutionary genetics analysis across computing platforms. Mol Biol Evol 35:1547-1549

Landmann F, Bain O, Martin C, Uni S, Taylor MJ, Sullivan W (2012) Both asymmetric mitotic segregation and cell-to-cell invasion are required for stable germline transmission of Wolbachia in filarial nematodes. Biol Open 1(6):536-47
Lefoulon E, Bain O, Makepeace BL, d'Haese C, Uni S, Martin C, Gavotte L (2016) Breakdown of coevolution between symbiotic bacteria Wolbachia and their filarial hosts. PeerJ 4:e1840

Lefoulon E, Clark T, Guerrero R, Cañizales I, Cardenas-Callirgos JM, Junker K, Vallarino-Lhermitte N, Makepeace BL, Darby AC, Foster JM, Martin C (2020) Diminutive, degraded but dissimilar: Wolbachia genomes from filarial nematodes do not conform to a single paradigm. Microb Genom 6(12):e000487

Lefoulon E, Clark T, Borveto F, Perriat-Sanguinet M, Moulia C, Slatko BE, Gavotte L (2020b) Pseudoscorpion Wolbachia symbionts: diversity and evidence for a new supergroup S. BMC Microbiol 20(1): $1-5$

Lefoulon E, Gavotte L, Junker K, Barbuto M, Uni S, Landmann F, Laaksonen S, Saari S, Nikander S, de Souza LS, Casiraghi M (2012) A new type F Wolbachia from Splendidofilariinae (Onchocercidae) supports the recent emergence of this supergroup. Int J Parasitol 42(11):1025-1036

Manoj RRS, Latrofa MS, Epis S, Otranto D (2021) Wolbachia: endosymbiont of onchocercid nematodes and their vectors. Parasit Vectors 14(1):1-24

Martin AR, Brown GK, Dunstan RH, Roberts TK (2005) Anaplasma platys: an improved PCR for its detection in dogs. Exp Parasitol 109(3):176-180

Martin C, Gavotte L (2010) The bacteria Wolbachia in filariae, a biological Russian dolls' system: new trends in antifilarial treatments. Parasite 17(2):79-89

Martinez J, Tolosana I, Ok S, Smith S, Snoeck K, Day JP, Jiggins FM (2017) Symbiont strain is the main determinant of variation in Wolbachia-mediated protection against viruses across Drosophila species. Mol Ecol 26(15):4072-4084

Mendoza-Roldan J, Ribeiro SR, Castilho-Onofrio V, Grazziotin FG, Rocha B, Ferreto-Fiorillo B, Pereira JS, Benelli G, Otranto D, Barros-Battesti DM (2020) Mites and ticks of reptiles and amphibians in Brazil. Acta Trop 208:105515

Mendoza-Roldan JA, Colella V, Lia RP, Nguyen VL, Barros-Battesti DM, Iatta R, Dantas-Torres F, Otranto D (2019) Borrelia burgdorferi (sensu lato) in ectoparasites and reptiles in southern Italy. Parasit Vectors 12(1):1-9

Mendoza-Roldan JA, Ravindran Santhakumari Manoj R, Latrofa MS, Iatta R, Annoscia G, Lovreglio P, Stufano A, Dantas-Torres F, Davoust B, Laidoudi Y, Mediannikov O, Otranto D (2021) Role of reptiles and associated arthropods in the epidemiology of rickettsioses: a one health paradigm. PLoS Negl Trop Dis 15(2):e0009090

Mendoza-Roldan JA, Mendoza-Roldan MA, Otranto D (2021b) Reptile vector-borne diseases of zoonotic concern. Int J Parasitol Parasites Wildl 15:132-142

Otranto D, Sakru N, Testini G, Gürlü VP, Yakar K, Lia RP, DantasTorres F, Bain O (2011) First evidence of human zoonotic infection by Onchocerca lupi (Spirurida, Onchocercidae). Am J Trop Med Hyg 84(1):55-58

Rasgon JL, Scott TW (2004) Phylogenetic characterization of Wolbachia symbionts infecting Cimex lectularius L. and Oeciacus vicarius Horvath (Hemiptera: Cimicidae). J Med Entomol 41(6): 1175-1178

Ros VI, Fleming VM, Feil EJ, Breeuwer JA (2009) How diverse is the genus Wolbachia? Multiple-gene sequencing reveals a putatively new Wolbachia supergroup recovered from spider mites (Acari: Tetranychidae). Appl Environ Microbiol 75(4):1036-1043

Shiny C, Krushna NS, Archana B, Farzana B, Narayanan RB (2009) Serum antibody responses to Wolbachia surface protein in patients with human lymphatic filariasis. Microbiol Immunol 53(12):685-693

Tamura K, Stecher G, Peterson D, Filipski A, Kumar S (2013) Molecular evolutionary genetics analysis version 6.0. Mol Biol Evol 30(12):2725-2729 
Taylor MJ, Hoerauf A (1999) Wolbachia bacteria of filarial nematodes. Parasitol Today 15(11):437-442

Taylor MJ (2003) Wolbachia in the inflammatory pathogenesis of human filariasis. Ann N Y Acad Sci 990(1):444-449

Werren JH, Windsor D, Guo LR (1995) Distribution of Wolbachia among neotropical arthropods. Proc Royal Soc B P Roy Soc B-Bol Sci 262(1364):197-204.

Zhou W, Rousset F, O'Neill S (1998) Phylogeny and PCR-based classification of Wolbachia strains using wsp gene sequences. Proc Royal Soc B P Roy Soc B-Bol Sci 265(1395):509-515.
Publisher's note Springer Nature remains neutral with regard to jurisdictional claims in published maps and institutional affiliations. 